possibly reflecting its availability as the only combination PI accessible in tablet/liquid form. Current use of PIs was associated $(p<0.001)$ with hypercholesterolaemia in adjusted analyses indicating a long-term consequence of specific ART.

Conclusions The majority of subjects had been managed with ART, with first exposure occurring at an early age. At least a quarter of participants had been treated with multiple individual drugs suggesting cumulative exposure and switching between regimens. The most prevalent treatment approach at recruitment was cART. However, there is evidence of continued use of suboptimal management strategies, and hypercholesterolaemia being associated with PI use.

\section{P24 A CLUSTER-RANDOMISED CONTROLLED TRIAL TO TEST THE EFFECTIVENESS OF A HAND WASHING INTERVENTION IN REDUCING INFECTION-RELATED ABSENCE IN PRIMARY SCHOOLS: INSIGHTS FROM AN EMBEDDED PROCESS EVALUATION}

doi:10.1136/jech.2010.120477.24

C R Chittleborough, R Campbell, A L Nicholson, S Gunn. Department of Social Medicine, University of Bristol, Bristol, UK

Objective To conduct a qualitative process evaluation within a cluster-randomised trial of an educational resource intervention to promote hand washing in primary schools and thus reduce absenteeism by reducing the transmission of respiratory and gastrointestinal infections.

Design Focus groups with pupils including drawings of hand washing facilities, semi-structured interviews with teachers, direct observation of intervention delivery and hand washing facilities.

Setting State primary schools within six local authority areas in the South West of England $(n=178)$ were randomised to receive the "Hands up for Max!" intervention in October 2009 (intervention schools) or in Autumn 2010 after all trial follow-up data are collected (control schools). Four intervention and four control schools were selected for the process evaluation from the 24 schools participating in a substudy to collect enhanced absenteeism data.

Participants Pupils in years 2 to $6(n=95)$, and key stage $1(n=8)$ and key stage $2(n=8)$ teachers.

Main Outcome Measures The process evaluation examined how the "Hands up for Max!" educational resource was delivered in intervention schools and explored responses to the intervention among pupils and staff. Ideas, attitudes, knowledge and behaviours relating to hand hygiene and hand washing facilities were explored, and hand washing facilities were observed in both intervention and control schools.

Results The "Hands up for Max!" resource was well received by the intervention schools, although some teachers made useful suggestions for improvements. Schools differed in the way they delivered the intervention and the number of elements of the resource package they used. Pupils in intervention schools recalled learning about the importance of hand washing in reducing the spread of infections and were able to describe, in detail, how to wash their hands properly. In the focus groups, pupils provided insight into reasons why they may not wash their hands, and what might help people wash their hands properly. Use of drawings in the focus groups facilitated discussion about what pupils liked and did not like about the facilities where they washed their hands. Results of the process evaluation were also used to inform development of questionnaires to obtain quantitative data from pupils and staff in all 178 schools participating in the trial.

Conclusion Information from the process evaluation will be useful in understanding any observed differences in quantitative outcomes related to absenteeism and knowledge, attitudes and behaviours related to hand washing, between intervention and control schools.
P25 ANTIBIOTIC PRESCRIBING IN IRISH CHILDREN RECEIVING FREE MEDICAL CARE

doi:10.1136/jech.2010.120477.25

${ }^{1} \mathrm{C}$ Keogh, ${ }^{1,2} \mathrm{U}$ Reulbach, ${ }^{1} \mathrm{~N}$ Motterlini, ${ }^{3} \mathrm{~K}$ Bennett, ${ }^{1} \mathrm{~T}$ Fahey. ${ }^{1}$ HRB Centre for Primary Care Research, Royal College of Surgeons Ireland, Dublin, Ireland; ${ }^{2}$ Public Health \& Primary Care, Trinity College Dublin, Dublin, Ireland; ${ }^{3}$ Pharmacology \& Therapeutics, Trinity College Dublin, Dublin, Ireland

Objective High rates of antibiotic prescribing are an increasing worldwide concern, in terms of increased antimicrobial resistance and associated burden to health services. However, there remains a lack of paediatric population-based pharmacoepidemiological research. The aim of the current study is to determine trends in systemic antibiotic prescribing in Irish children.

Design Retrospective analysis of routinely collected data.

Participants Data were obtained from the General Medical Services (GMS) claims database. This represents patients who are unable to pay for medical services and accounts for about one third of Irish children. Data were obtained from 2004 to 2008, for about 271000 children per year aged $\leq 15$.

Main Outcome Antibiotic prescribing behaviour in a paediatric population.

Results Prescribing rates of systemic antibiotics were compared across years, age $(0-4,5-11,12-15)$ and gender and are presented with CI and significance values (using negative binomial regression analysis). In 2004, 631/1000 population (95\% CI 628 to 634) received at least one antibiotic prescription compared to $578 / 1000$ ( $95 \%$ CI 575 to 581) in 2008. However, the overall trend across the 5 -year period did not show a significant change (IRR 0.99, 95\% CI 0.96 to $1.0, p=0.23)$. No significant difference was observed between males $(620 / 1000,95 \%$ CI 618 to 622$)$ and females $(631 / 1000,95 \%$ CI 629 to 633 ; IRR 1.02, 95\% CI 0.97 to $1.06, \mathrm{p}=0.46$ ). However, prevalence rates significantly decreased with age. Children in the 0-4 age group were significantly more likely to receive an antibiotic prescription $(827 / 1000,95 \%$ CI 824 to 829$)$, relative to the 5-11year old $(553 / 1000,95 \%$ CI 552 to 556$)$ and $12-15$ years $(489 / 1000$ $95 \%$ CI 487 to 492 ), with all $p<0.001$. The net ingredient cost of antibiotics increased from $€ 2.3$ million in 2004 to $€ 2.8$ million in 2008. The five most commonly prescribed drugs were as follows: amoxicillin (298/1000, 95\% CI 297 to 299), co-amoxiclav (296/1000, 95\% CI 295 to 296), cefaclor (135/1000, 95\% CI 134 to 136), clarithromycin (54/1000, 95\% CI 53 to 54 ) and phenoxymethylpenicillin (53/1000, 95\% CI 52 to 53). The overall rates observed here are significantly higher than those reported elsewhere in Europe. For example, the Netherlands (178/1000), Denmark (328/1000) and Scotland $(142 / 1000)$ consistently report low antibiotic prescribing rates. The choice of agents also differed between countries.

Conclusions Prescribing rates remained stable over the 5 -year period. Although the rates observed here are higher than European comparisons, these results should be considered in the context of the GMS population. Nevertheless, the results suggest the possible overuse of antibiotics within the GMS population and the potential benefit from interventions to reduce prescribing.

\section{Older people \\ P26 INTERVENTIONS TO IMPROVE PRESCRIBING QUALITY IN CARE HOMES: A SYSTEMATIC REVIEW}

doi:10.1136/jech.2010.120477.26

${ }^{1} \mathrm{M}$ Loganathan, ${ }^{1} \mathrm{~S}$ Singh, ${ }^{1}$ Alex Bottle, ${ }^{2} \mathrm{~B}$ D Franklin, ${ }^{1} \mathrm{~A}$ Majeed. ${ }^{1}$ Department of Primary Care and Public Health, Imperial College London, London, UK; ${ }^{2}$ Centre for Medication Safety and Service Quality, Imperial College Healthcare NHS Trust/The School of Pharmacy, University of London, London, UK

Introduction Prescribing in the elderly population is a complex process and the prevalence of inappropriate prescribing is high, with 
potentially severe consequences. Care home residents are particularly vulnerable to inappropriate prescribing. With a growing ageing population, strategies to improve prescribing are essential. The aim of this systematic review was to collect and interpret the results of controlled trials of interventions to reduce inappropriate prescribing in care homes, to determine the most effective strategies. Method Databases searched were MEDLINE, EMBASE, international pharmaceutical abstracts and the Cochrane library. Search items included "nursing home", "residential home", "inappropriate prescribing", "education", "staff education", "MDT", "pharmacist", "computer". The search strategy retrieved 16 articles that met the inclusion criteria. Two independent reviewers undertook screening and methodological quality assessment, using the Downs and Black rating scale. A meta-analysis could not be done due to heterogeneity of the outcome measures used in the different studies.

Results Four intervention strategies were indentified: education, multidisciplinary team (MDT) meetings, clinical pharmacist reviews and computerised clinical support systems. Educations interventions was the most studied area, with six studies showing an improvement in inappropriate prescribing. Mixed results were found for the pharmacist interventions, possibly due to the inappropriate choice of outcome measures used for assessing prescribing quality. Computerised decision support systems were evaluated in two studies, with one showing a significant increase in the final appropriate drug orders. Two of the three studies examining MDT meetings found an overall improvement in quality of prescribing.

Conclusion Results from various interventional strategies are mixed; a multi-faceted approach, clearer policy guidelines and standardised measurements for measuring inappropriate prescribing are required to improve prescribing practices for these vulnerable patients.

\section{P27 OLDER ADULTS WITH CANCER-ARE THOSE WHO LIVE ALONE AT THE END OF LIFE A DISADVANTAGED GROUP? A QUALITATIVE STUDY}

doi:10.1136/jech.2010.120477.27

\begin{abstract}
${ }^{1} \mathrm{~L}$ Cooper, ${ }^{2} \mathrm{~J}$ Addington-Hall, ${ }^{3} \mathrm{~A}$ Arthur, ${ }^{4} \mathrm{G}$ Grande, ${ }^{5} \mathrm{~S}$ Payne, ${ }^{3} \mathrm{~J}$ Seymour, ${ }^{1} \mathrm{~B}$ Hanratty. ${ }^{1}$ Division of Public Health, University of Liverpool, Liverpool, UK; ${ }^{2}$ School of Health Sciences, University of Southampton, Southampton, UK; ${ }^{3}$ School of Nursing, Midwifery \& Physiotherapy, University of Nottingham, Nottingham, UK; ${ }^{4}$ School of Nursing, Midwifery \& Social Work, University of Manchester, Manchester, UK; ${ }^{5}$ Division of Health Research, Lancaster University, Lancaster, UK
\end{abstract}

Health status will help to determine where an older adult can live, but it is also possible that living arrangements may themselves have consequences for the health experience of older adults towards the end of life. This study will explore the relevance to cancer patients of factors that the literature suggests might account for poor health and different patterns of service use amongst solitary living older people.

Objective To determine how older adults with cancer, living alone with advanced disease, have distinct experiences, needs and preferences for care, which merit attention.

Design In-depth qualitative interviews with 30 people, half of whom live alone. Participants were recruited from general practices and day hospices in the North West, aged over 75 years; with a documented diagnosis of cancer and professionally determined prognosis of less than 12 months. Baseline face-to-face interviews were followed by telephone contacts at 3 and 6 months. Data were analysed using Framework, a matrix-based approach.

Findings Many of the older adults in this study described substantial networks of intergenerational, spousal and neighbour support, irrespective of living arrangements. Those who lived alone had high levels of functioning and quality of life despite poor health status and practical challenges. The desire to maintain independence in all aspects of their lives was a key theme. Loneliness and social isolation were not common, with few differences observed with living arrangements. Companion animals played an important social role and helped to define the daily routine for some participants who lived alone.

Conclusion Living arrangements are easily observed, and a convenient way of defining a population for study. However, the availability of familial and community support may be a more important influence on older people's end of life experiences. Our findings suggest that the very old living alone with cancer may be a particularly resilient group of people.

\section{P28 PATTERNS OF DIETARY SUPPLEMENT USE AMONG OLDER MEN AND WOMEN: FINDINGS FROM THE HERTFORDSHIRE COHORT STUDY}

doi:10.1136/jech.2010.120477.28

${ }^{1} \mathrm{H}$ J Denison, ${ }^{1} \mathrm{~S} M \mathrm{M}$ Robinson, ${ }^{1} \mathrm{~K}$ A Jameson, ${ }^{1} \mathrm{H}$ E Syddall, ${ }^{1} \mathrm{H} \mathrm{J}$ Martin,

${ }^{1} \mathrm{E}$ M Dennison, ${ }^{1} \mathrm{C}$ Cooper, ${ }^{1,2} \mathrm{~A}$ Aihie Sayer. ${ }^{1} \mathrm{MRC}$ Epidemiology Resource Centre, University of Southampton, Southampton, UK; ${ }^{2}$ Academic Geriatric Medicine, University of Southampton, Southampton, UK

Setting Use of dietary supplements has increased over recent decades in the UK. Although dietary supplement use has been described in a number of populations we know little about patterns of supplement use in older people.

Objective The objectives of this study were to describe dietary supplement use in the Hertfordshire Cohort Study (HCS), to determine patterns of supplement use, and to investigate the extent to which supplement user groups differ in terms of their characteristics, diets and morbidity.

Methods 3217 HCS participants, aged 59 to 73, were interviewed. Diet over the preceding 3 months was assessed by FFO; compliance with "healthy" eating recommendations was defined using individual scores for a "prudent" dietary pattern, identified using principal components analysis. Details of the brand and dose of all dietary supplements taken in the preceding 3 months were recorded. Individual supplements were allocated to one of 10 groups based on their nutrient composition, and cluster analysis was used to define groups of supplement users. The demographic and lifestyle characteristics, and morbidity of the supplement user groups was examined.

Results $45.4 \%$ of men and $57.5 \%$ of women reported taking at least one dietary supplement in the previous 3-month period; the most commonly taken type of supplement was oils (43\% of total supplement products taken), particularly fish liver oil. There were five distinct clusters of supplement users; these were common to men and women. They were labelled according to the principal supplement taken; oils, glucosamine, single vitamins, vitamins and minerals, and herbal products. For men, there were differences between the groups for BMI $(p=0.030)$, "prudent" diet score $(p=0.002)$, Hospital Anxiety and Depression score $(p=0.013)$ and social class $(p=0.012)$. For women, there were differences between the groups for age $(p=0.030)$, "prudent" diet score $(p=0.014)$ and social class $(p=0.005)$. There was no significant difference between the groups for Hospital Anxiety and Depression score among the women. With the exception of a difference in diagnosis of diabetes among the women $(p=0.021)$, there were no differences in morbidity between the supplement groups in either men or women.

Conclusion There were distinct patterns of supplement use in the HCS. Supplement user groups differed in their characteristics, but there were few differences in morbidity. 